\title{
Inclusion of authorized deception in the informed consent process does not affect the magnitude of the placebo effect for experimentally induced pain
}

\author{
Andrea L. Martin *, Joel Katz \\ Department of Psychology, Behavioral Sciences Building, York University, 4700 Keele Street, Toronto, Ont., Canada M3J 1P3
}

\begin{abstract}
A B S T R A C T
The ethics of placebo research have been of paramount concern since the discovery of the phenomenon. To address these ethical concerns, Miller and colleagues (PLoS Med 2005 Sep;2(9):e262, 0853-0859) propose an alternate approach to placebo research, called "authorized deception", in which participants are alerted of the use of deception in the research prior to study enrollment and thus knowingly permit its use if they decide to participate. The present study sought to investigate the authorized deception methodology in experimentally induced placebo analgesia. The participants were randomly assigned to an authorized deception or non-authorized deception group. A commonly used protocol was employed wherein heat pain stimulation was surreptitiously lowered following the application of a placebo cream during a series of conditioning trials and the magnitude of the placebo effect was subsequently assessed in test trials for which the stimulus intensity was the same for both the placebo and control creams. Authorized deception did not have any negative impact on the magnitude of the placebo effect, recruitment and retention of participants, nor did it result in any significant psychological harm. The majority of participants who received this form of consent preferred it to the traditional approach in which the participants are not alerted to the presence of deception. These findings suggest that the use of authorized deception is a viable and ethically preferable alternative consent process for laboratory-based studies on placebo analgesia. Further studies are needed to examine the effect of authorized deception in clinical trials and other placebo research within a clinical setting.
\end{abstract}

\section{Introduction}

Placebos have the potential to enhance the therapeutic outcome of medical interventions and are a source of important variability to be considered in clinical trials designed to evaluate treatment efficacy [14]. However, the methodology by which placebo effects are investigated has raised some ethical concerns $[5,10,14,15]$. Because placebo analgesia is strongly influenced by the recipient's expectations [16-18], clinical and experimental studies typically rely on the use of deception regarding the purpose of the research and/or the nature of the treatment being administered, thereby leading the participants to believe that they are receiving a physically active treatment when in fact they are receiving a placebo [14]. This use of deception is often justified on the grounds that full disclosure about the purpose of the research or the experimental procedures may influence the participants' responses and thus jeopardize scientific knowledge [14]. As such, deception is often seen as a necessary means of promoting the internal and external validities of placebo research [13].

\footnotetext{
* Corresponding author. Tel.: +1 $4167362100 \times 33125$

E-mail address: amartin@yorku.ca (A.L. Martin).
}

However, as Miller and colleagues [13,15] highlight, use of deception conflicts with ethical principles of human experimentation as it: (1) violates the principle of respect for persons by failing to disclose relevant information that might affect an individual's decision to volunteer for a research study; (2) may manipulate individuals to participate in research that they would not have; and (3) may cause distress and lack of trust in research when the deception is revealed. Furthermore, the American Pain Society position statement on the use of placebos in clinical pain management highlights, "deception of patients about clinical treatments violates the right of patients to consent to or refuse treatment" ([22] p. 216).

Miller and colleagues $[14,15,26]$ advocate an alternate approach to the consent process in deceptive research. The participants are informed, prior to deciding whether to participate in a study that some features of the study will or may be misleading or deceptive. They call this form of consent "authorized deception", since the participants are alerted to the presence of deception in the research and thus knowingly permit its use if they decide to participate [15]. Additionally, the participants may be offered the opportunity to withdraw their data after they have been informed about the true nature of the study as a means of restoring participant autonomy [14]. 
Criticisms of the authorized deception approach are that it may create suspicion, thus resulting in biased data [15], reduce participant enrollment [2,15] and/or cause anxiety [4]. Providing participants the opportunity to withdraw their data also may limit the generalizability of research findings [26] and introduce a participation bias.

The present study was designed to provide an evidence-based evaluation of the authorized deception methodology in experimentally induced placebo analgesia. The participants were randomly assigned to an authorized deception group or a nonauthorized deception group. Key outcome variables included assessment of differences in participant enrollment, magnitude of the placebo effect, mood, satisfaction with the research experience, attitudes toward researchers, beliefs regarding respect/infringement of individual rights, and willingness to participate in future research of this kind.

\section{Methods}

\subsection{Participants}

Participants comprised 40 adults ( 28 females and 12 males; mean age $=21.18, S D=3.34 \mathrm{yrs}$ ) recruited from flyers posted around the University campus. Prospective participants underwent an initial telephone screen to rule out any medical conditions or medication use that might interfere with pain sensitivity or increase risk of unnecessary discomfort during thermal sensory testing. Individuals were excluded if they reported an ongoing pain problem, high blood pressure, circulatory problems, diabetes, heart disease, asthma, seizures, frostbite, past trauma to the hands or arms, lupus, other large or small joint disease or injury, or use of analgesics, anti-inflammatory medications, psychoactive drugs and/or antihistamines. The York University Research Ethics Board reviewed and approved the study protocol. The participants received $\$ 20$ for their involvement in the study.

\subsection{Apparatus}

\subsubsection{Experimental setting}

The experiment took place on campus in a room set up with medical equipment to resemble a physician's office including an examination table, privacy curtain, medical scale for measuring height and weight, blood pressure cuff, metal equipment tray, containers of cotton balls, tongue depressors, plastic syringes, rubbing alcohol, and wall posters depicting the musculoskeletal system, symptoms of neuropathic pain and the pathophysiology and anatomy of arthritis and knee injury.

\subsubsection{Medoc thermal sensory analyzer}

Heat pain was induced by means of a Medoc TSA-II thermal stimulator (Ramat Yishai, Israel). The TSA-II is a computerized device designed to measure sensory thresholds to vibration and temperature (e.g., warm, cold, heat-induced pain and cold-induced pain). The TSA-II is used in a variety of clinical disorders (e.g., diabetes, peripheral neuropathy) to obtain a quantitative evaluation of the integrity of both small (A-delta and $C$ ) and large diameter (A-beta) sensory nerve fibers. The TSA-II is capable of delivering thermal stimuli that range from approximately $0^{\circ} \mathrm{C}$ to $50^{\circ} \mathrm{C}$. A thermode is attached to the participant's skin with a Velcro strap and heat stimuli of various temperatures are administered. A participant-initiated button press stops the rise in temperature and the thermode rapidly returns to room temperature at a rate of $\sim 4^{\circ} \mathrm{C} / \mathrm{s}$. In the present study, thermal stimuli of $5 \mathrm{~s}$ duration were applied to the skin through a thermode with a contact area $3 \mathrm{~cm}^{2}$. The temperature of the thermode rose rapidly $\left(4^{\circ} \mathrm{C} / \mathrm{s}\right)$ from a base- line temperature of $35^{\circ} \mathrm{C}$ to a pre-programmed peak temperature where it remained for $5 \mathrm{~s}$ before returning to baseline.

\subsubsection{Placebo cream}

The same over-the-counter hypoallergenic moisturizer cream (Glaxal Base), which does not contain an active analgesic agent, was used for both the placebo cream and the control cream. The creams were visible in two plastic syringes on a metal medical tray with the labels 'Alevocaine ${ }^{\mathrm{TM}}$ ' and 'Control Cream'. The experimenter wore latex gloves while handling, applying, and removing the creams. The creams were removed with an alcohol swab.

\subsection{Response measures}

\subsubsection{Numeric rating scale (NRS) - pain}

The participants were asked to rate the intensity of pain stimuli using an 11-point, self-report, numeric rating scale (NRS) [7] ranging from 0 to 10 , with endpoints representing no pain $(0)$ and the most intense pain imaginable (10). The participants were asked to choose the number that best corresponded to the intensity of each heat pain stimulus they received. The NRS provides a simple, efficient and minimally intrusive measure of pain intensity. This scale is commonly used in clinical settings [1] and is the preferred pain rating scale among patients [27]. The NRS is highly correlated $(r=0.94)$ with the visual analog scale [1] and is sensitive to change following pharmacological interventions [7].

\subsubsection{Profile of mood states (POMS)}

The profile of mood states (POMS) [12] is a widely used, 65-item self-report scale designed to measure affective mood states and their fluctuations in medical patients, psychiatric outpatients, normal adults, and college students. Each item is rated on a 5-point scale ranging from not at all (0) to extremely (4). The right now rating period was used for the present study. The POMS yields a total mood disturbance (TMD) score and 6-factor analytic-derived scales including; (1) tension-anxiety, (2) depression-dejection, (3) anger-hostility, (4) vigor-activity, (5) fatigue-inertia, and (6) confusion-bewilderment. The POMS has excellent internal consistency $(\alpha=0.84-0.95)$ and fair test-retest reliability $(r=0.43-0.53)$ over a 6 -week period in patients receiving psychiatric treatment [11]. Evidence of the predictive and construct validity of the POMS has been demonstrated in brief psychotherapy research, cancer research, controlled outpatient drug trails, studies on sports and athletes, and studies of response to emotion-inducing conditions in healthy populations [11].

\subsubsection{Numeric rating scale (NRS) - attitudes}

A numeric rating scale (ranging from 0 to 10 ) was used to assess participants' attitudes across four domains: (1) satisfaction with the research experience, (2) feelings toward researchers who plan and run studies such as the present one, (3) the extent to which they felt that their individual rights were respected while taking part in the study, and (4) their willingness to participate in similar research in the future.

\subsection{Procedure}

The participants were randomly assigned to one of two groups using the randomization program available at www.randomization.com: authorized deception group or non-authorized deception group. An experimenter wearing a white lab coat greeted the participants upon arrival for the study. The participants were provided with a consent form, and the experimenter verbally described the study following a standard script. The participants in both groups were told that the investigators were examining the effectiveness of a new topical anesthetic cream called Alevocaine ${ }^{\mathrm{TM}}$ which had 
been shown to reduce pain in some individuals; and that the Alevocaine $^{\mathrm{TM}}$ cream would be compared against a regular moisturizer cream. The details of the thermal stimulator and the method of assessing the effectiveness of the cream by means of painful heat stimuli were described and the participants were told that they could discontinue participation in the study at any time without negative consequences.

The authorized deception group and the non-authorized deception group received identical information in the consent form with the exception of one paragraph added to the end of the consent form for the authorized deception group. In this paragraph, prospective participants were informed, prior to deciding whether to volunteer for the study, that some aspects of the study were being intentionally misdescribed. Specifically, the following statement, recommended by Miller et al. [15], was included at the end of the consent form for the authorized deception group:

"You should be aware that the investigators have intentionally misdescribed certain aspects of the study. This use of deception is necessary to obtain valid results. However, an independent ethics committee has determined that this consent form accurately describes the major risks or benefits of the study. The investigators will explain the misdescribed aspects of the study to you at the end of your participation" (p. 856).

The experimenter also repeated this statement verbally when reviewing the study procedures with the participant to ensure that all participants in the authorized deception group were aware of this information.

Following the consent procedures, the participants in both the authorized deception and non-authorized deception groups completed the POMS and a demographic and pain history questionnaire. The participants were then brought into a laboratory room set-up to resemble a physician's office and underwent four consecutive stages of thermal sensory testing involving a set of familiarization trials, calibration trials, conditioning trials, and test trials [19].

\subsubsection{Familiarization trials}

In order to familiarize participants with the range of temperatures, one trial each of $44^{\circ}, 45^{\circ}, 47^{\circ}$, and $49^{\circ} \mathrm{C}$ stimuli was delivered in ascending order on the ventral side of the participant's right forearm.

\subsubsection{Calibration trials}

The participants then underwent a series of calibration trials similar to that of Price and colleagues [19] to control for individual differences in pain perception. A series of 16 thermal stimuli ranging between $44^{\circ}$ and $49^{\circ} \mathrm{C}$ was administered in a random order and the participants were asked to rate the pain intensity of each stimulus on a $0-10$ NRS. At the end of the calibration trials, a regression equation was calculated for each participant in order to predict thermal intensity (temperature in ${ }^{\circ} \mathrm{C}$ ) from verbal pain intensity report (NRS pain ratings). This calculation was used to determine the temperature corresponding to each individual's NRS pain rating of 6 and 3, which varied among participants depending on their own personal perception of pain. These two stimulus levels (i.e., temperatures) were used in all subsequent trials and were specific to each individual.

\subsubsection{Conditioning trials}

A plastic template was used to mark two squares on the ventral side of the participant's right forearm. Two square adhesive patches with the center cut out were applied to these two areas to demarcate the two locations on the arm where the creams were
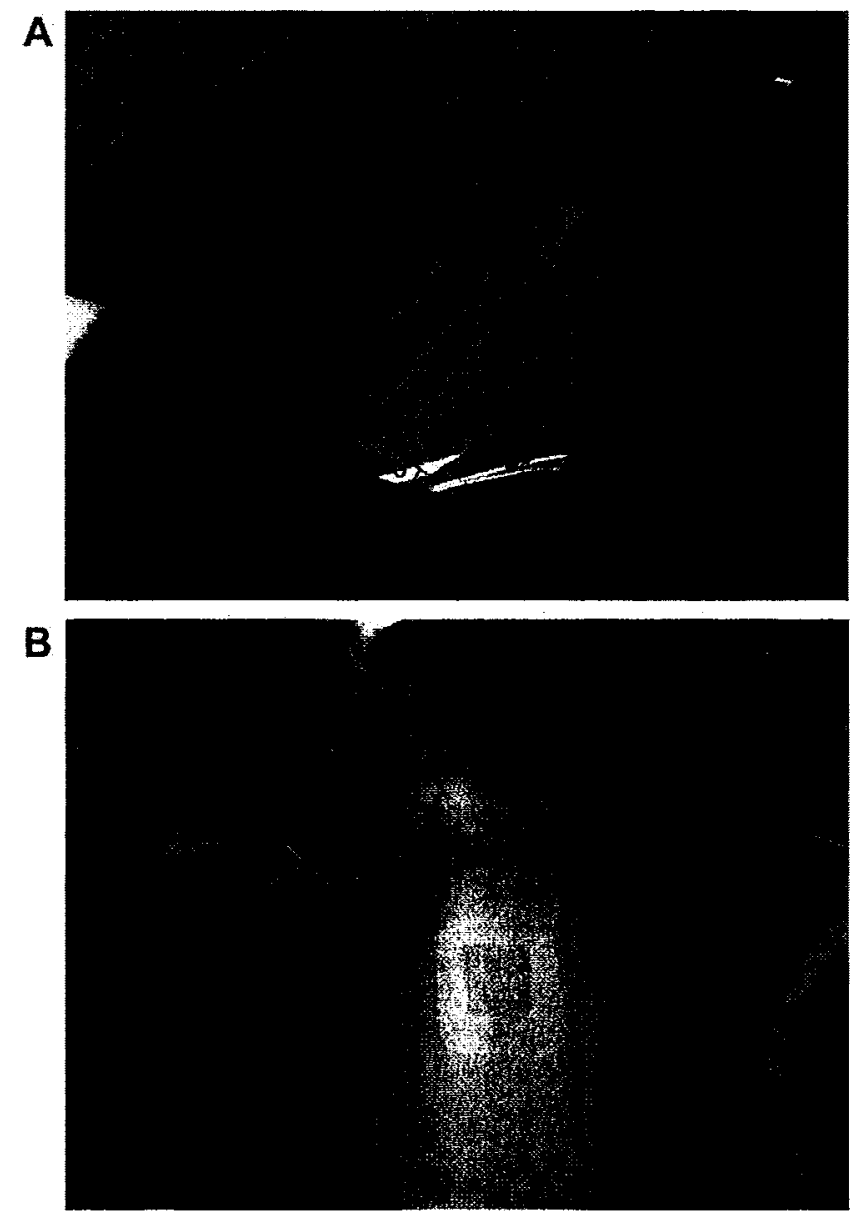

Fig. 1. (A) Plastic template used to mark two squares on the ventral side of the participant's right forearm. (B) Adhesive patches used to demarcate the two locations on the arm where the creams were applied.

applied (Fig. 1). In line with previous research [17,23-25], a conditioning procedure was used in which the intensity of heat pain stimuli was surreptitiously lowered during conditioning trials for the placebo cream (i.e., Alevocaine ${ }^{\mathrm{TM}}$ cream). That is, in order to create the impression of analgesic efficacy (i.e., pain relief) when testing in the area of skin where the placebo cream was applied the temperature was surreptitiously lowered to a level corresponding to the participant's NRS pain rating of 3 . When testing in the area of skin where the control cream was applied, the temperature was administered at a level corresponding to the participant's NRS pain rating of 6 . The participants were asked to verbally rate the intensity of each stimulus using the 0-10 NRS. One block of four thermal stimuli was administered for each cream at each of the two locations according to a randomized counterbalanced design such that each participant received eight conditioning trials for each cream.

\subsubsection{Test trials}

Immediately following the conditioning trials, the participants received one final test trial with each cream. The stimulus intensity for the test trials was the same for both the placebo (Alevocaine ${ }^{\mathrm{TM}}$ ) and the control creams. That is, for the test trial, the stimulus intensity was raised to a temperature corresponding to a pain rating of 6 for the 'Alevocaine ${ }^{\mathrm{TM}}$ ' condition. The magnitude of the placebo analgesic effect was determined by comparing the test trial pain ratings for the placebo versus the control cream. 


\subsection{Post-test interview and debriefing}

Following the test trials and before the debriefing process, the participants were asked a series of questions about their feelings concerning the research procedures they had just undergone and their thoughts about the Alevocaine ${ }^{\mathrm{TM}}$ cream. The participants in the authorized deception group were asked to describe how they had felt when they read in the consent form that certain aspects of the study were being intentionally misdescribed and that the use of deception was necessary to obtain valid results. They were also asked if they wondered what aspect(s) of the study had been misdescribed and if so, what these aspects might have been.

The participants in both the authorized deception and nonauthorized deception groups were asked "At any point during the study: did you wonder if Alevocaine ${ }^{\mathrm{TM}}$ had side effects that we weren't telling you about; did you wonder if Alevocaine ${ }^{\mathrm{TM}}$ was another drug that we didn't tell you about; and, did you wonder if Alevocaine ${ }^{\mathrm{TM}}$ might not be a real drug?" If the participants answered yes to any of these questions they were asked "When did you start to wonder this?" "Did this thought make you feel more anxious?" and "Did this thought influence your pain ratings during the study? (And if so, how?)"

Following this set of questions, the participants were given a debriefing form describing the true purpose of the study and the nature of the placebo cream. In the debriefing form the participants were also offered the opportunity to withdraw their data from the study if they felt concerned or uncomfortable about the fact that they had been intentionally deceived. When given the debriefing form, the participants in the authorized deception group were told, "As you are aware, there were certain aspects of this study that I was not able to explain fully to you at the beginning of your participation. I would like to give you a debriefing form now that will explain the purpose of the study and outline the aspects that I was not able to tell you about at the beginning". The participants in the non-authorized deception group were told, "There were aspects of this study that I was not able to explain fully to you at the beginning of your participation. I would like to give you a debriefing form now that will explain the purpose of the study and outline the aspects that I was not able to tell you about at the beginning".

Immediately after the participants finished reading the debriefing forms they were asked to complete the POMS a second time to assess any changes in mood following their participation in the study and the debriefing procedures. A brief set of questions was then administered to both groups to assess their attitudes about the research experience (see Section 2.3.3). Additionally, the participants in the authorized deception group were re-read the same paragraph from the consent form alerting them to the presence of deception in the study and were asked, "Having participated now, would you rather not have been informed of this at the beginning of the study?" The participants in the non-authorized deception group were read this same paragraph and were asked, "Having participated now, would you rather have been informed of this [i.e., alerted to the presence of deception] at the beginning of the study?"

\section{Results}

\subsection{Magnitude of the placebo effect}

The magnitude of the placebo effect for each group was calculated by subtracting the pain intensity rating for the placebo cream from the pain intensity rating for the control cream during the final trial (i.e., when the temperature was the same for both creams). The resulting placebo response score represents the change in pain

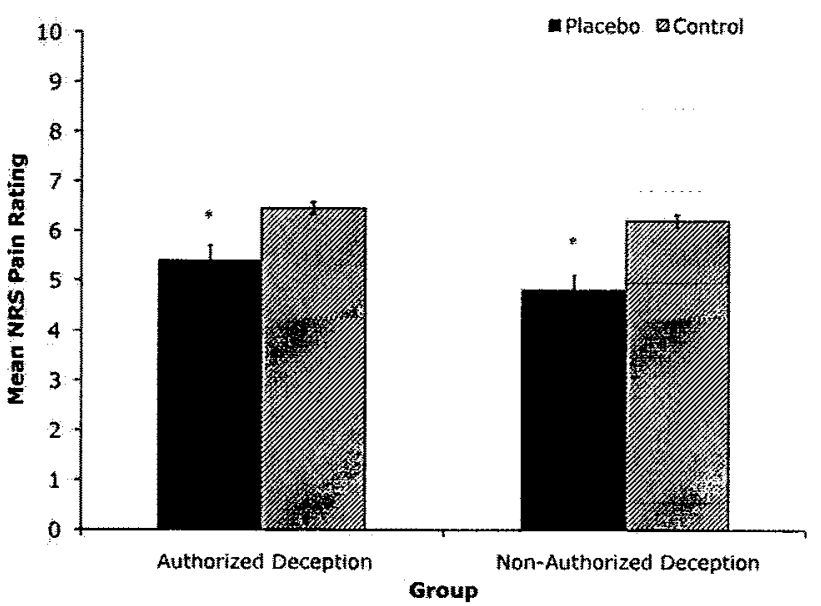

Fig. 2. Mean pain ratings for the placebo (Alevocaine ${ }^{\mathrm{TM}}$ ) and control creams in the authorized deception and non-authorized deception groups. Error bars represent standard errors. Data indicate a significant placebo effect for each group. $p<0.001$ comparing placebo (Alevocaine ${ }^{\mathrm{TM}}$ ) with control.

intensity rating due to the administration of the placebo [17]. Both the authorized deception and the non-authorized deception groups obtained positive scores averaging $1.05(S D=1.43)$ and 1.40 $(S D=2.35$ ), respectively, indicating a less painful response to the placebo cream than to the control cream (i.e., a placebo effect).

In order to determine whether there was a difference in the magnitude of the placebo effect between the authorized deception group and the non-authorized deception group, a $2 \times 2$ betweenwithin analysis of variance (ANOVA) was conducted, using group (authorized deception and non-authorized deception) as the between-subjects factor and cream (placebo and control) as the within-subjects repeated measures factor. ANOVA results revealed a non-significant group $\times$ cream interaction, $F(1,38)=0.32$, $p=0.57$. The main effect of cream (Placebo/Control), $F$ $(1,38)=15.87, p<0.001$, indicated significantly lower pain ratings for placebo $(M \pm S D=5.10 \pm 2.50$ ) as compared to control cream $(M \pm S D=6.33 \pm 2.09)$ across both groups. The group effect was not significant, $F(1,38)=0.41, p<0.53$. Taken together, these results indicate that both groups showed a significant placebo effect, the magnitude of which did not differ significantly between the authorized deception and non-authorized deception groups (Fig. 2).

\subsection{Mood}

Means and standard deviations for each of the POMS scales are presented in Table 1. A series of between-within ANOVAs were conducted on each of the following POMS scales using time (post-consent vs. post-debrief) as the within-subjects factor and group (authorized deception and non-authorized deception) as the between-subjects factor: (1) tension-anxiety, (2) depressiondejection (3) anger-hostility, (4) vigor-activity, (5) fatigue-inertia, (6) confusion-bewilderment, and (7) total mood disturbance. Neither the group main effect nor the group $\times$ time interaction effect was significant for any of the mood scales. The time effect revealed significant improvements from post-consent to post-debrief for all the mood scales, with the exception of the vigor-activity scale, which remained unchanged (Table 2). These results indicate significant improvements, independent of group, over time for tensionanxiety, depression-dejection, anger-hostility, fatigue-inertia, confusion-bewilderment, and total mood disturbance. 
Table 1

Means and standard deviations of POMS scores for authorized deception and non-authorized deception groups at time 1 (post-consent) and time 2 (post-debriefing).

\begin{tabular}{|c|c|c|c|c|}
\hline \multirow[t]{2}{*}{ POMS scale } & \multicolumn{2}{|c|}{ Authorized deception group } & \multicolumn{2}{|c|}{ Non-authorized deception group } \\
\hline & Time $1 M(S D)$ & Time $2 M(S D)$ & Time $1 M(S D)$ & Time $2 M(S D)$ \\
\hline$T-A$ & $5.45(3.68)$ & $4.05(2.48)$ & $6.60(3.76)$ & $4.15(2.08)$ \\
\hline $\mathrm{D}-\mathrm{D}$ & $4.80(7.58)$ & $1.7(3.88)$ & $535(10.05)$ & $3.20(6.86)$ \\
\hline$A-H$ & $2.45(3.66)$ & $1.35(2.37)$ & $4.30(8.29)$ & $2.00(3.31)$ \\
\hline$V=A$ & $11.80(6.99)$ & $12.35(10.92)$ & $11.70(5.93)$ & $11.15(5.63)$ \\
\hline $\mathrm{FAI}$ & $5.15(4.58)$ & $3.50(3.49)$ & $6.50(5.46)$ & $4.45(4.59)$ \\
\hline $\mathrm{C}-\mathrm{B}$ & $5.65(3.57)$ & $3.75(2.40)$ & $5,70(3,63)$ & $3.95(2.98)$ \\
\hline TMD & $11.70(22.18)$ & $2.00(18.17)$ & $16.75(30.24)$ & $6.60(19.66)$ \\
\hline
\end{tabular}

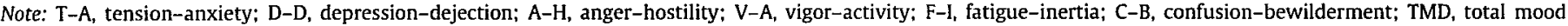
disturbance.

\subsection{Attitudes}

A multivariate analysis of variance was conducted to examine group differences across the four attitude variables: (1) satisfaction with the research experience, (2) feelings toward researchers who plan and run studies like this, (3) the extent to which the participants felt that their individual rights were respected while taking part in the study, and (4) willingness to participate in research of this kind in the future. Overall, the participant ratings averaged eight (out of 10) or greater for each of the four attitude variables indicating a high degree of satisfaction with their experience in the study, positive feelings toward researchers, feeling that their individual rights had been respected and a high degree of willingness to participate in future research of this kind. Significant group differences were not observed for any of the four attitudes' variables (Table 3).

\subsection{Enrollment, withdrawal from the study, and feelings about deception}

Study enrollment, retention of the participants, and withdrawal of the data were not affected by the authorized deception procedure (Fig. 3). Specifically, none of the prospective participants in the authorized deception group decided against participating in the study after learning in the consent form that they would be deceived if they were to participate in the study. Similarly, all prospective participants in the non-authorized deception groups agreed to participate in the study after reading the informed consent form. No participants from either group withdrew from the study and none of the participants in either group took up our offer to remove their data from the study after the debriefing process.

At the end of the study, just prior to the debriefing process, the participants in the authorized deception group were asked how they felt when they read in the consent form that certain aspects of the study had been intentionally misdescribed and that this use of deception was necessary to obtain valid results. Almost all the participants (90\%) reported feeling curious and intrigued upon hearing that certain aspects of the study had been intentionally misdescribed. Few subjects reported experiencing negative reactions such as feelings of anxiety (30\%), uncertainty (10\%) and anger (5\%). Most participants (85\%) reported wondering what parts of the study had been misdescribed to them.

Chi-squared analysis did not reveal a significant difference in the proportion of the participants in the authorized deception (9/ 20 ) and non-authorized deception (7/20) groups who wondered if Aleovcaine ${ }^{\mathrm{TM}}$ might not be a real drug, $\chi^{2}(1)=0.42, p=0.52$. The stage of the experiment at which these participants wondered about the veracity of the Alevocaine ${ }^{\mathrm{TM}}$ is presented in Table 4. Six participants in the authorized deception group reported wondering if Alevocaine ${ }^{\mathrm{TM}}$ might not be a real drug during the actual experiment; however, these numbers did not differ from those in the non-authorized deception group where five participants also reported wondering about the veracity of the Alevocaine ${ }^{\mathrm{TM}}$ while the creams were being tested. These results suggest that any suspicion about the true nature of the creams was likely not due to the fact that the authorized deception group had been alerted to the presence of deception in the study.

At the end of the study, following the debriefing, the paragraph that was included in the consent form for the authorized deception group, alerting them to the presence of deception in the study, was read to all participants. The participants in the non-authorized deception group were asked, "Having participated now, would you rather have been informed of this?" while the participants in the authorized deception group were asked, "Having participated now, would you rather not have been informed of this?" In the non-authorized deception group, three participants reported that they would rather have been informed of the deception at the outset of the study and one of these participants stated that she would be more cautious about future research as a result of her experience with the deception in this study (Table 5). However, 17 participants in the non-authorized deception group reported that they would not rather have been informed of the deception at the outset of the study. Of these 17, 10 felt that knowing about the deception would have made them suspicious, thus biasing their responses and negatively impacting the outcome of the study, and seven stated that they did not have a strong preference for one set of

Table 2

POMS between-within ANOVA results with group (authorized deception and non-authorized deception) and time (post-consent and post-debrief).

\begin{tabular}{|c|c|c|c|}
\hline POMS scale & Time $1 M(S D)$ & Time $2 M(S D)$ & Time 1-time 2 change (within-subjects effect) \\
\hline Tension-anxiety & $6.03(3.72)$ & $4.10(2.26)$ & $F(1,38)=21.04, p<0.001$ \\
\hline Depression-dejection & $5.08(8.79)$ & $2.45(5.56)$ & $F(1,38)=15.86, p<0,001$ \\
\hline Anger-hostility & $3.38(6.40)$ & $1.68(2.86)$ & $F(1,38)=5.71, p=0.02$ \\
\hline Vigor-activity & $11.75(6.40)$ & $11.75(8.60)$ & $F(1,38)=0.00, p=1.00$ \\
\hline Fatigue-inertia & $5.83(5.02)$ & $3.98(4.05)$ & $F(1,38)=18.88, p<0.001$ \\
\hline Confusion-bewilderment & $5.68(3.55)$ & $3.85(2.68)$ & $F(1,38)-31.34, p<0.001$ \\
\hline Total mood disturbance & $14.23(26.30)$ & $4.30(0.39)$ & $F(1,38)=23.90, p<0.001$ \\
\hline
\end{tabular}

Note; "Time 1", post-consent; "Time 2", post-debrief; "Time 1" and "Time 2" represent average scores on each scale after collapsing across groups. 
Table 3

Means, standard deviations and MANOVA results for attitude variables across the study groups.

\begin{tabular}{|c|c|c|c|}
\hline 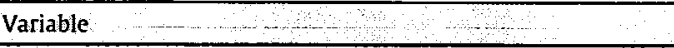 & Authorized deception $M(S D)$ & Non-authorized deception $M(S D)$ & Between-group differences \\
\hline Satisfaction with their research experience & $8.45(1.00)$ & $7.85(1.84)$ & $F(1,38)=1.64, p=0.21$ \\
\hline $\begin{array}{l}\text { Feelings toward researchers who plan and run studies } \\
\text { like this, involving deception }\end{array}$ & $8.60(1.43)$ & $8.45(1.54)$ & $F(1,38)=0.10, p=0.75$ \\
\hline $\begin{array}{l}\text { Extent to which they felt that their individual rights were } \\
\text { respected throughout their participation in the study }\end{array}$ & $9.65(0.81)$ & $9.70(0.57)$ & $F(1,38)=0.05, p=0.82$ \\
\hline Willingness to participate in research of this kind in the future & $9.40(1.31)$ & $8.65(1.53)$ & $F(1,38)=2.76, p=0.11$ \\
\hline
\end{tabular}

instructions over the other or felt that it was more interesting to find out at the end.

In contrast, 19 out of 20 participants in the authorized deception group reported that they would have preferred to be alerted, as they had been, to the presence of deception in the study. Upon further questioning, of these 19 participants, 13 expressed a clear preference to have been alerted to the deception and seven of these 13 stated they would have experienced a negative reaction to the study, such as anger, disappointment, and feeling as though they had been cheated had they not been warned about the use of deception (Table 5). The other six of these 19 participants stated that they did not have a strong preference for one set of instructions over the other. The one participant who reported a preference not to have known about the deception felt that the research find-

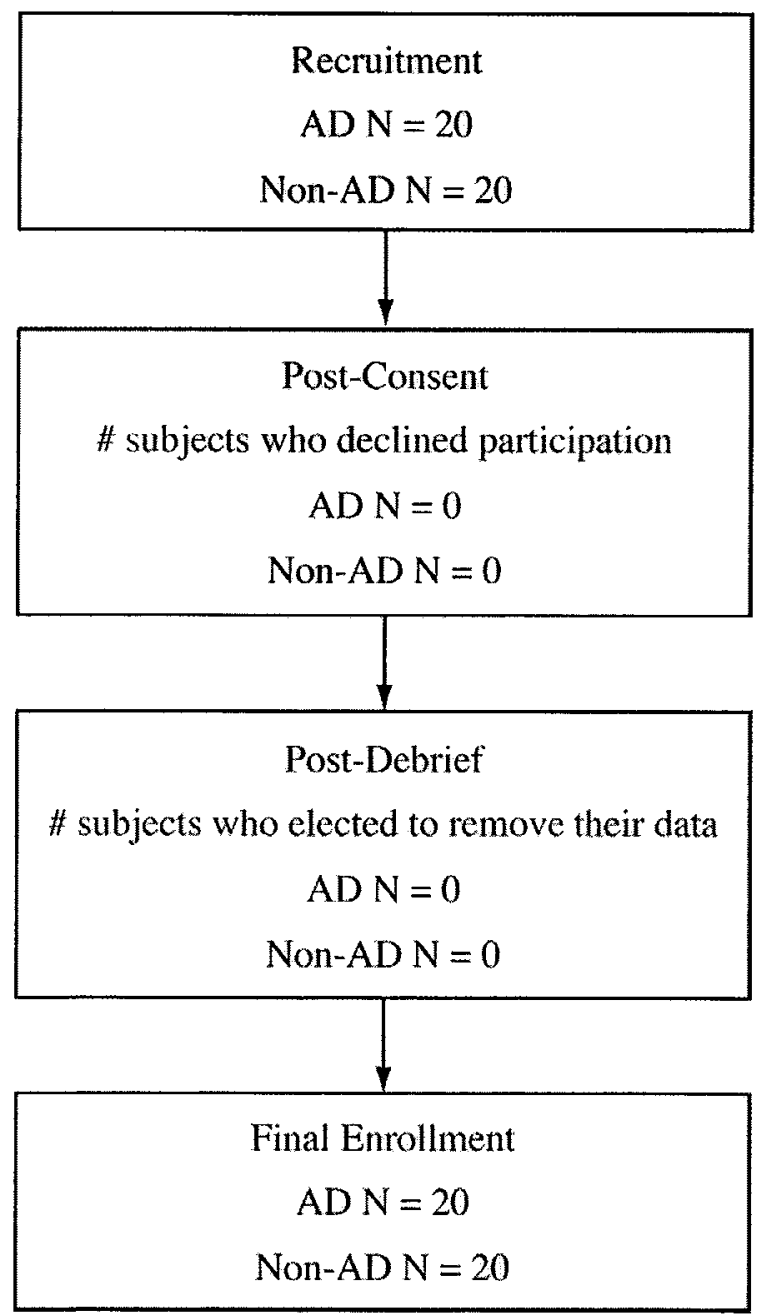

Fig. 3. Flow-chart depicting participant recruitment and final enrollment for the two groups. $A D$, authorized deception group; non- $A D$, non-authorized deception group. ings would be more accurate if the participants were not warned about the use of deception.

\section{Discussion}

The present study sought to investigate an alternate approach to participant consent in placebo research involving the use of deception. Authorized deception is designed to alert the participants to the presence of deception in the research, without informing them about the exact nature of the deception [13-15,26]. The results of the present study show that the magnitude of the placebo effect did not differ significantly between the participants who were informed about the presence of deception and those who were not. While it is possible that some degree of temporal summation occurred across study trials such that pain increased with subsequent thermal stimuli [6] this would have occurred in both study groups and thus should not account for any differences, or lack thereof, between the authorized deception and the nonauthorized deception groups.

Authorized deception has been proposed as a more ethical approach to placebo research since it allows the participants the opportunity to decide whether or not they are willing to volunteer for a study that involves deception [15]. Although authorized deception does not replace full informed consent, it is designed to promote the autonomy of research participants by giving them a fair opportunity to withdraw from a study knowing that deception will be involved [14]. Another measure to promote autonomy in deceptive research is to provide the participants the opportunity to withdraw their data after having learned the true nature of the study during the debriefing process [14].

The results of the present study do not support the concerns that authorized deception has adverse effects on key outcome variables, thus compromising the scientific validity of the data and introducing bias. Important indicators of whether the use of authorized deception is a viable alternative to the traditional approach of informed consent (i.e., non-authorized deception) in placebo research of this nature include potential detrimental effects on recruitment and retention of participants as well as participants' decisions to withdraw their data following debriefing. The present results indicate that use of authorized deception did not have a negative impact on any of these measures; enrollment and retention of the participants were identical between the two groups

Table 4

Phase of experiment at which participants in each group retrospectively reported wondering if Alevocaine ${ }^{\mathrm{TM}}$ was not a real drug.

\begin{tabular}{l|ll}
\hline Phase of experiment & AD & Non-AD \\
\hline Before coming into the study & 2 & 1 \\
While reading the consent form & 1 & 0 \\
While completing the questionnaires at the beginning & 1 & 0 \\
While the creams were being tested & 4 & 5 \\
Now (i.e., when asked) & 1 & 1 \\
Total \# of subjects & 9 & 7 \\
\hline
\end{tabular}

Note: $\mathrm{AD}$, authorized deception group; non-AD, non-authorized deception group. 
Table 5

Comments from participants in the authorized deception and non-authorized deception groups expressing a negative reaction to the use of deception.

\begin{tabular}{|c|c|}
\hline Group & Comments \\
\hline AD & "I would have been angry if there was deception and you didn't tell me" \\
\hline AD & "It's good to know labout the deception], If not I would have been angry, I was in another study with deception and they didn't tell me and I felt angry" \\
\hline $\mathrm{AD}$ & "[1] would be angry" \\
\hline AD & $\begin{array}{l}\text { I'm very glad you had that clause in there at the beginning so I could ask my questions and feel okay about participating I would have wondered why you } \\
\text { didn't warn me otherwise" }\end{array}$ \\
\hline $\mathrm{AD}$ & "I would feel cheated if you hadn't told me" \\
\hline $\mathrm{AD}$ & $\begin{array}{l}\text { "[1] would prefer to know about the deception. It made me feel more comfortable. If you didn't tell me I would have feit disappointed and more anxious at } \\
\text { the end, wondering, what else didn't they tell me?" }\end{array}$ \\
\hline $\mathrm{AD}$ & "I would have been disappointed if you hadn't told me" \\
\hline Non-AD & "It's better to know as much as you can. I would be more cautious about future research. I would do it but l'd be looking for deception" \\
\hline
\end{tabular}

and none of the participants in the present study chose to withdraw their data when given the option to do so after the debriefing process (Fig. 2).

There is little literature regarding participant decisions to withdraw data when offered the opportunity [13]. However, the present results are in line with at least one other study which examined the placebo response in relation to asthma in a clinical population and found none of the 55 patients accepted the offer to withdraw their data [8]. The present findings support the use of authorized deception and granting participants the option to withdraw their data as two methods of enhancing the ethical principles of placebo research without compromising the scientific validity and generalizability of the research findings.

The results of the present study also do not support the suggestion that alerting subjects to the use of deception results in increased anxiety [4]. While a few participants reported experiencing some anxiety upon hearing aspects of the study had been intentionally misdescribed, the vast majority ( $90 \%$ ) reported feeling curious and intrigued. Furthermore, there were no differences in mood between the two groups (as measured by the POMS and verbal self-report) following the debriefing when the true nature of the creams and the purpose of the study were revealed.

We hypothesized that the non-authorized deception group would demonstrate a worsening of mood relative to the authorized deception group following the debriefing since the former group had not been warned about the use of deception in the study. We therefore expected these participants to report a negative reaction to the realization that what they thought was an active drug was actually a placebo. However, contrary to this expectation, both groups exhibited improvements in mood across the study. These findings are similar to those of Chung and colleagues [3] who found reductions in anxiety, frustration and fear after revealing to the participants the magnitude of their placebo response in relation to experimentally induced pain.

These improvements in mood may represent an artifact of repeat testing or may reflect participants' relief at having completed the study [3]. Positive interactions with the experimenter throughout the study may have also contributed to improvements in mood. One limitation of the present study is the lack of a true baseline measure of mood prior to the authorized deception intervention. This would have allowed for an assessment of mood changes resulting from the authorized deception intervention; however, given that the intervention involved altering the informed consent process and therefore took place before consent had been obtained, from an ethical standpoint, it was not possible to obtain a prior baseline assessment of mood.

Another potential concern with the use of authorized deception is that it may create suspicion in research participants, thus resulting in biased data [15]. Although a small proportion of the participants in the authorized deception group questioned the true nature of the Alevocaine ${ }^{\mathrm{TM}}$ cream, this was no greater than that of the non-authorized deception group, suggesting that any suspi- cion about the authenticity of the cream was not related to the authorized deception manipulation. Suspicion, expressed by some participants, about the authenticity of the cream could have been related to various factors; including, not experiencing a noticeable reduction in pain when the Alevocaine ${ }^{\mathrm{TM}}$ cream was applied, or the fact that the study was advertised as a 'Health Psychology' study and as a primarily undergraduate population, research participants may have been familiar with psychological research involving deception [13].

Interestingly, 13 participants in the authorized deception group expressed a clear preference to be alerted to the presence of deception in the study compared to only three participants in the nonauthorized deception group. Several explanations may account for these findings. It is possible that the participants did not have strong feelings about the use of deception in the research. To the extent that this is true, it is important to note that this may be specific to the present population, which consisted predominantly of healthy undergraduate students who we would not expect to have a vested interest in the outcome of the study or the efficacy of the 'drug' under investigation.

Other studies have found that the use of deception in psychological research is not distressing to many subjects $[20,21]$; however, healthy undergraduate students may have different attitudes toward being deceived than patients participating in a clinical trial [9]. The degree to which an individual is troubled by deception may depend on the extent to which the deceived relies on the deceiver [26]. As such, the present findings may not generalize to clinical populations who are likely more vulnerable to deception, often placing considerable faith and trust in clinical investigators.

It is also possible that the participants in the non-authorized deception group downplayed the negative effects they experienced as a result of the deception and thus our findings may not have accurately captured their true feelings. Participants may, as a means of 'saving face' [26], minimize the effects of deception or choose not to report them, particularly when they are asked by the very experimenter who has just deceived them. This explanation may also account for the finding that more than a third of participants in the authorized deception group reported that they would have experienced a negative reaction had they not been warned about the use of deception, while none of the participants in the non-authorized deception group reported actually experiencing such a reaction. It is also possible that the prior warning about the use of deception may have engendered a greater sense of partnership with and trust in the experimenters, thus allowing the authorized deception group to more openly share any negative feelings.

Other data from the authorized deception group also support the use of the authorized deception methodology in placebo research. Over half the participants in this group reported that they preferred to be warned about the use of deception at the outset of the study, prior to agreeing to participate. Numerous partici- 
pants expressed gratitude for the openness with which they were informed of the deception and some participants reported that they would have likely felt some anger or resentment had they not been warned (Table 5 ).

\section{Conclusions}

Taken together, the results of the present study suggest that alerting participants to the presence of deception in experimental pain studies on placebo analgesia does not affect the magnitude of the placebo effect, recruitment and retention of participants, nor does it result in any significant psychological harm. Indeed, the majority of participants who received this form of consent preferred it to the traditional approach of not alerting participants to the presence of deception. These findings support the use of authorized deception in laboratory-based studies of placebo analgesia and suggest that it is a viable and ethically preferable method of informed consent compared to the traditional approach. Studies are needed to examine the effect of authorized deception in clinical trials and other placebo research within a clinical setting.

\section{Acknowledgments}

Andrea Martin is supported by a Canadian Institutes of Health Research (CIHR) Doctoral Award. Joel Katz is supported by a CIHR Canada Research Chair (CRC) in Health Psychology at York University. The study was funded by a CIHR CRC in Health Psychology and by infrastructure grants from the Canadian Foundation for Innovation and the Ontario Innovation Trust. This paper is derived, in part, from Andrea Martin's Doctoral Dissertation. The authors do not have any conflicts of interests associated with this manuscript.

\section{References}

[1] Bijur PE, Latimer CT, Gallagher EJ. Validation of a verbally administered numerical rating scale of acute pain for use in the emergency department. Acad Emerg Med 2003;10:390-2.

[2] Boter H, van Delden JJM, de Haan RJ, Rinkel GJE. Patients' evaluation of informed consent to postponed information: cohort study. $\mathrm{Br}$ Med J 2004;329:86.

[3] Chung SK, Price DD, Verne GN, Robinson ME. Revelation of a personal placebo response: its effects on mood, attitudes and future placebo responding. Pain 2007; $132: 281-8$
[4] Dawson AJ. Methodological reasons for not gaining prior informed consent are sometimes justified. Br Med J 2004;329:87.

[5] Evans M. Justified deception? The single blind placebo in drug research. J Med Ethics 2000;26:188-93.

[6] Granot M, Granovsky Y, Sprecher E, Nir R. Yarnitsky D. Contact heat-evoked temporal summation: tonic versus repetitive-phasic stimulation. Pain 2006;122:295-305.

[7] Katz J, Melzack R. Measurement of pain. Surg Clin North Am 1999;79:231-52.

[8] Kemeny ME, Rosenwasser LJ, Panettieri RA, Rose RM, Berg-Smith SM, Kline JN. Placebo response in asthma: a robust and objective phenomenon.JAllergy Clin Immunol 2007;119:1375-81.

[9] Korn JH. Judgments of acceptability of deception in psychological research. J Gen Psychol 1987:114:215-6.

[10] Kotzalidis GD, Pacchiarotti I, Manfredi G, Savonja V, Torrent C, Mazzarini L, Tatarelli C, Amann B, Di Marzo S, Sanchez-Moreno J, Sani G, Girardi P, Colom F, Vieta E. Ethical questions in human clinical psychopharmacology: should the focus be on placebo administration? J Psychopharmacol 2008;22:590-7.

[11] McNair DM, Heuchert JWP. Profile of mood states: technical update. North Tonawanda, NY: Multi-Health Systems Inc.; 2003.

[12] McNair DM, Lorr M, Droppleman LF. Manual: profile of mood states. San Diego, CA: Educational and Industrial Testing Service; 1971.

[13] Miller FG, Gluck JP, Wendler D. Debriefing and accountability in deceptive research. Kennedy Inst Ethics J 2008;18:235-51.

[14] Miller FG, Kaptchuk T]. Deception of subjects in neuroscience: an ethical analysis. J Neurosci 2008;29:4841-3.

[15] Miller FG, Wendler D. Swartzman LC. Deception in research on the placebo effect. PLoS Med 2005;2.

[16] Montgomery G, Kirsch I. Mechanisms of placebo pain reduction: an empirical investigation. Psych Sci 1996;7:174-6.

[17] Montgomery GH, Kirsch I. Classical conditioning and the placebo effect. Pain 1997:72:107-13.

[18] Price DD, Finniss DG, Benedetti F. A comprehensive review of the placebo effect: recent advances and current thought. Annu Rev Psychol 2008;59:565-90.

[19] Price DD, Milling LS, Kirsch I, Duff A, Montgomery GH, Nicholls SS. An analysis of the factors that contribute to the magnitude of placebo analgesia in an experimental paradigm. Pain 1999;83:147-56.

[20] Seber JE, Iannuzzo R, Rodriguez B. Deception methods in psychology: have they changed in 25 years? Ethics Behav 1995;5:67-85.

[21] Soliday E, Stanton AL. Deceived versus nondeceived participants' perceptions of scientific and applied psychology. Ethics Behav 1995:87-104.

[22] Sullivan M, Terman GW, Peck B, Correll DJ, Rich B, Clark WC, Latta K, Levbovits A, Gebhart G. APS position statement on the use of placebos in pain management. J Pain 2005;6:215-7.

[23] Voudouris N. Peck C, Coleman G. Conditioned placebo responses. J Pers Soc Psychol 1985;48:47-53.

[24] Voudouris N, Peck C, Coleman G. Conditioned response models of placebo phenomena: further support. Pain 1989;38:109-16.

[25] Voudouris N, Peck C, Coleman G. The role of conditioning and verbal expectancy in the placebo response. Pain 1990;43:121-8.

[26] Wendler D, Miller FG. Deception in the pursuit of science. Arch Intern Med 2004;164:597-600.

[27] Williamson A, Hoggart B. Pain: a review of three commonly used pain rating scales. J Clin Nurs 2005:14:798-804 